\title{
AIRBORNE IMAGING OF TROPOSPHERIC EMISSION AT MILLIMETER AND SUBMILLIMETER WAVELENGTHS
}

\author{
A.J. Gasiewski and D.M. Jackson \\ School of Electrical and Computer Engineering \\ Georgia Institute of Technology \\ Atlanta, GA 30332-0250 \\ J.R. Wang and P.E. Racette \\ NASA Goddard Space Flight Center \\ Greenbelt, MD 20771

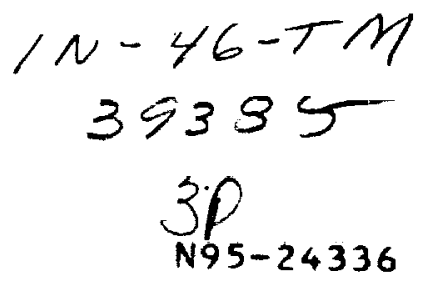

D.S. Zacharias

ZAX Millimeter Wave Corporation San Dimas, CA 91773

Unclas

\section{G3/46 0039385}

\section{ABSTRACT}

In September 1993, the NASA Millimeter-wave Imaging Radiometer (MIR) few on board the NASA ER-2 high-altitude aircraft during $C A M E X$, and obtained the first wideband millimeterand submillimeter-wavelength images of tropospheric emission. The MIR is a cross-track scanning radiometer with chandels at $89.150,183 \pm 1,3,7,220$, and $325 \pm 1,3,8 \mathrm{GHz}$. This set provides upwelling brightness information at the two strong rotational water vapor lines at 183.310 and $325.153 \mathrm{GHz}$ and three nearby atmospheric transmission windows. The wideband MIR images of convective raincells reveal unique cloud and precipitation map. ping capabilities that are not available from lower frequency $\mathrm{mi}$ crowave channels. Comparisons between the 183 and $325 \mathrm{GHz}$ spectra also reveal differential brightness temperature modes that are related to cloud water.

\section{INTRODUCTION}

Satellite-based imaging of atmospheric clouds and water vapor has been successfully performed using both microwave (e.g.. the DMSP SSM/I and SSM/T-2 microwave instruments and the NOAA MSU instrument) and IR and visible channels (e.g., the visible and IR imagers on the NOAA geostationary and polar orbiting satellites). Historically, frequencies between the microwave and IR bands have received little at tention for at least two reasons: (1) The development of tecbnology for sensitive radiometess at millimeter-wave (MMW) and submillimeter-wave (SMMW) $\mathrm{fr}$ quencies bas lagged such developments at both the lower microwave and visible/IR bands and (2) The opacity caused by water vapor and clouds is relatively large at MMW and SMMW frequencies and bas prompted concern that practical nadir imag. ing of the tropospheric meteorological phenomena would be pre. cluded. However, as discussed by Gasiew'ski (1992), the potential benefits of satellite-based MMW and SMMW radiometry include high spatial resolution using antennas of practical size and additional spectral information related to the underlying cloud and water vapor state. Accordingly, an investigation into tropospheric imaging using MMW and SMMW bands is underway. In this paper, we report the development and successful deployment of the first wideband MMW and SMMW radiometer for tropospheric cloud, precjpitation, and water vapor imaging.

The instrument used is the Millimeter-Wave Imaging Radiometer (MIR), developed by the NASA Goddard Space Flight Center, Georgia Institute of Technology, and ZAX Millimeter Wave Corporation (Racette et al., 1994). The MIR is a totalpower cross-track scanning radiometer with channels at 89,150 , $183 \pm 1,3,7,220$, and $325 \pm 1,3,8 \mathrm{GHz}$. The instrument is configured for flight on the NASA ER-2 high-altitude aircraft. All channels are linearly polarized and have identical $3.5^{\circ} 3-\mathrm{dB}$ beamwidths resulting in $1.2-\mathrm{km}$ spots on the surface at nadir. Crosstrack sampling and scan rates are set to provide nadir spatial ampling at $\sim 82 \%$ of the Nyquist rate or more than twice the commonly used $3-\mathrm{dB}$ spatial sampling rate. Detection is accomplished using five waveguide-fed mixers with integrated antennas. Each mixer is pumped by a Gunn diode local oscillator; both fundamental and harmonic mixers are used. The sensitivity for $\sim 30 \mathrm{msec}$ integration time is $\sim 0.2-0.3 \mathrm{~K}$ for the 89 . through $220-\mathrm{GHz}$ channels and $\sim 1.5 \mathrm{~K}$ for the $325-\mathrm{GHz}$ channels. Calibration is accomplished using pyramidal hot and cold blackbody standards. Absolute accuracy is $\sim 1-2 \mathrm{~K}$ for all channels.

The MIR was deployed on the ER-2 during CAMEX (the Convective Atmospheric Moisture Experiment), September-October 1993, during which all nine MMW and SMMW channels were operational. Flights over non-precipitating clouds, convective precipitation (both continental and maritime), and clear-air yielded the first wideband MMW and SMMW tropospheric imagery. This wideband MIR imagery is used here to illustrate several innova. tive and beneficial applications of high-resolution satellite-based MMW and SMMW observations. These include improvements in measurements of tropospheric water vapor profiles, cloud ice in measurements of tropospell extent. The imagery shows that SMMW channels provide unique information pertaining to water vapor density and cloud parameters, either when used alone or in conjunction with lower frequency microwave channels.

\section{CLEAR-AIR SPECTRAL FEATURES}

Calculations by Gasiewski (1992) suggested that clear air spectral variations in channels near the 183.310 and $325.153 \mathrm{GHz}$ water vapor lines should be quite similar; bence, spectral differences between these bands should be largely independent of water vapor variations. This hypothesis was investigated by comparing clear-air spectral differences observed during different fights and thus, for different water vapor states. The spectral differences are defined by.

$$
\Delta T_{183-325} \triangleq T_{183}-\bar{T}_{325}
$$

where:

$$
\begin{aligned}
& T_{183}=\left[T_{183 \pm 1}, T_{103 \pm 3}, T_{183 \pm 7}, T_{150}\right] \\
& \cdot T_{325}=\left[T_{325 \pm 1}, T_{325 \pm 3}, T_{325 \pm 8}, T_{220}\right]
\end{aligned}
$$

Clear-air data from two fights (sorties \#93-164 on September 17,1993 and \#93-168 on September 26, 1993) were examined to check for this behavior (Tablel). The average clear-air brightness changes between $9 / 17 / 93$ and $9 / 26 / 93$ ranged from $8-16 \mathrm{~K}$ for the channels being considered. However, the spectral differences between the 183 - and $325-\mathrm{GHz}$ bands varied by no more than $5.5 \mathrm{~K}$, with typical variations of $\sim 1 \mathrm{~K}$. With the exception of the $183 \pm 1-325 \pm 1$ difference the data show exceptional rejection of water vapor variations. The relatively large variation $(60 \%)$ in $T_{183 \pm 1}-T_{325 \pm 1}$ is partly due to noise in the $325 \pm 1-\mathrm{GHz}$ channel. 
Table 1: Clear-air brightness variations between flights on $9 / 17 / 93$ and $9 / 26 / 93$

\begin{tabular}{c|cc|cc}
\hline \hline & \multicolumn{2}{|c|}{$\bar{T}_{183-325}$} & \multicolumn{2}{c}{ Difference } \\
& $9 / 17 / 93$ & $9 / 26 / 93$ & $(9 / 17 / 93-9 / 26 / 93)$ \\
& $(\mathrm{K})$ & $(\mathrm{K})$ & $(\mathrm{K})$ & $(\mathrm{K})$ \\
\hline \pm 1 & -4.0 & -9.6 & 5.5 & 60.0 \\
\pm 3 & 9.3 & 8.7 & 0.6 & 4.7 \\
\pm 7 or \pm 8 & 17.5 & 16.2 & 1.3 & 8.4 \\
150 or 220 & 1.1 & 1.2 & -0.1 & 0.9 \\
\hline
\end{tabular}

It has also been suggested (Gasiewski 1992) that the large separation in frequency between the 18.3- and $325-\mathrm{GHz}$ bands (approximately one octave) should result in significantly different reproximately one ociare sarticles. Thus, clouds could be detected and distinguished from water vapor variations by thresholding the $\Delta \bar{T}_{183-325}$. To this end, we have demonstrated a raincell and cloud detector using the $183.325 \mathrm{GHz}$ spectral differences that is cloud detector to the underlying water vapor state. An optimized version of such a dual-band detector would be extremely useful in removing 1 he effects of thin clouds on $183-\mathrm{GHz}$ water vapor retrievals.

\section{CLOUD AND PRECIPITATION MAPPING}

Strip-map images of isolated convective precipitation cells over the Allantic ocean are shown in Fig. 1. Although the 32.5 over the channels are somewhat noisy, the capability to map raincells iu thic band witl, high contrast can be clearly seen. The low brightness temperat ures (as low as $130 \mathrm{~K}$ ) over cell cores are the result of scaltering of the cold cosmic background flux from ice particles in the cell top. Mans weak cells and clouds that escape particles in the cellop at $89 \mathrm{GHz}$ are clearly outlined ir. the $32.5-\mathrm{GHz}$ channels, and to a lesset extent in the 183 . and $220-\mathrm{GHz}$ channels.
The responses of the 183-, 220-, and 325-GHz channels to clouds and raincells are monotonic under the prevailing summer midlatitude humidity conditions and ocean surface state. The high zenith opacity of water vapor, low ocean reflectivity. and hig values of cloud scattering and absorption at 183, 220. and high values coling signature. This is unlike the $89 . \mathrm{GHz}$ channel (and to some extent, the $150 . \mathrm{GHz}$ channel), which exhibits a bimodal response to maritime clouds. Thin clouds cause a warming at $89 \mathrm{GHz}$ by increasing the opacity of the reflected path to cold space; heavy clouds and raincells with ice canopies cause strong scattering of the cold background flux. Although reflectivities of dense ice canopies at frequencies near $\sim 100 \mathrm{GHz}$ can exceed 60\% (Gasiewski and Staelin, 1990). many thin clouds are undetectable at $89 \mathrm{GHz}$.

Fig. 2 is a scatterplot of the nadir brightness values in Fig. 1 for the 89 . and $325 \pm 8-\mathrm{GHz}$ channels. The $325 \pm 8-\mathrm{GHz}$ values are for the $i$ and the clear-air value of $\sim 254$. $k$. The non-monotonic behavior of the $89 . \mathrm{GHz}$ channel results in a distribution of points behavior of the terplots show no evidence of saturation of the $325-\mathrm{GHz}$ channels even at the cell cores. The apparent lack of saturation suggests that these channels can provide a wide dynamic bright ness lem. perature range for purposes of cloud and raincell detection and perature range for purther, the large bright ness variations (up to $\sim 150 \mathrm{~K}$ ) in the $325-\mathrm{GHz}$ channels indicate that these channels to $\sim 150 \mathrm{~K}$ ) in the $32 . \mathrm{s}$ sitivity to clouds and raincells having moderate $(\sim 5-7 \mathrm{~km})$ top altitudes. As expected for spectral line imagery. the amplitude of the cloud-top scattering signature in the $325 . \mathrm{GHz}$ channels in creases as the channel frequency shifts away from the line center.

\section{WIDEBAND RAINCELL SPECTRA}

Raincells are best observed using radiometric channels centered in the atmospheric transmission window's. Although storm intensity and rain rate are correlated with MMW and SMMW

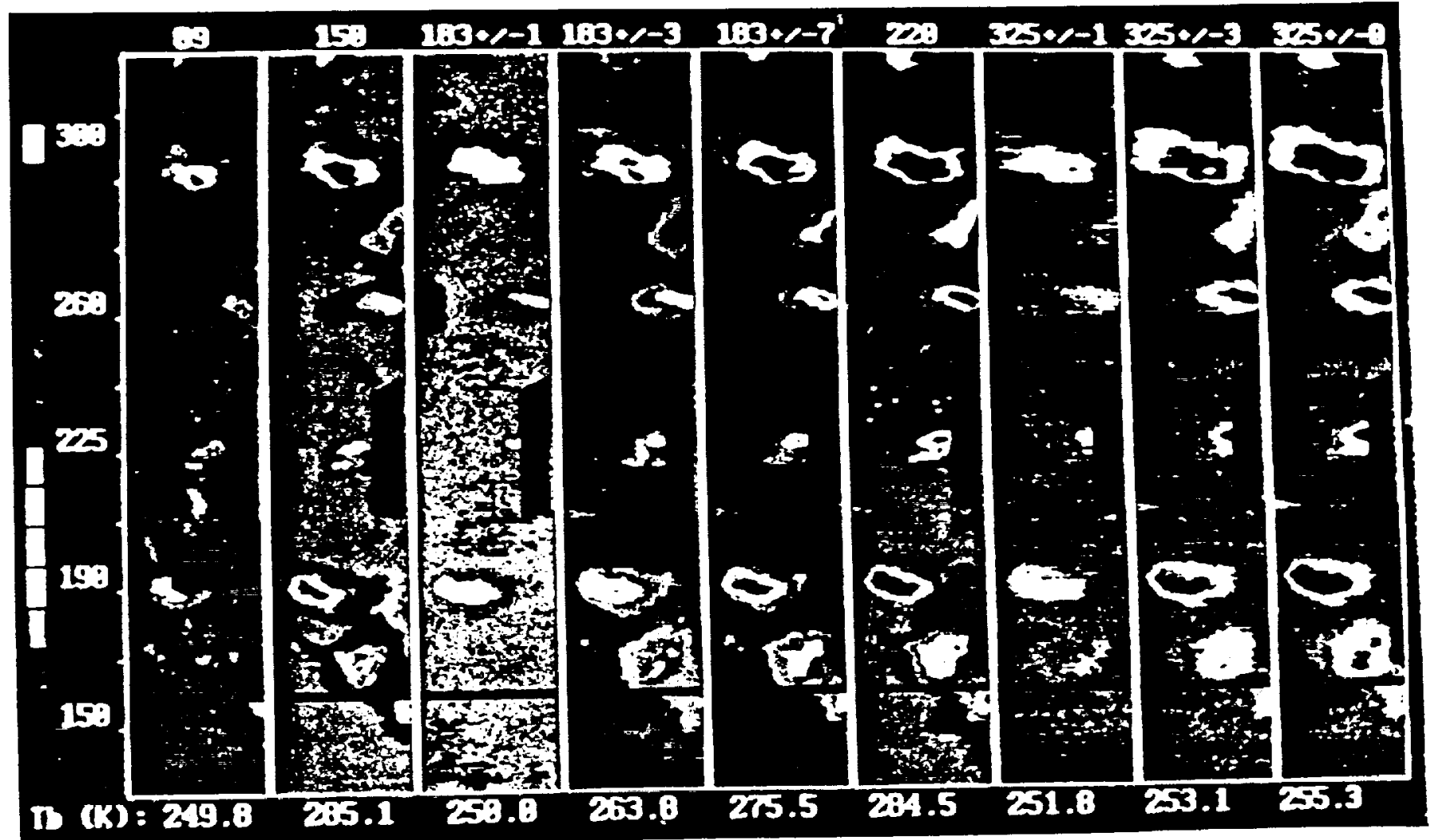

Figure 1: Strip map images of upwelling thermal emission from isolated convective cells over ocean (Seplember 27. 1994). Each map covers approximalely $40 \times 220 \mathrm{~km}$; cells are distorted slightly due their proximity to the aircraft. Average clear-air brightness temperature values at nadir are indicated below each map. 


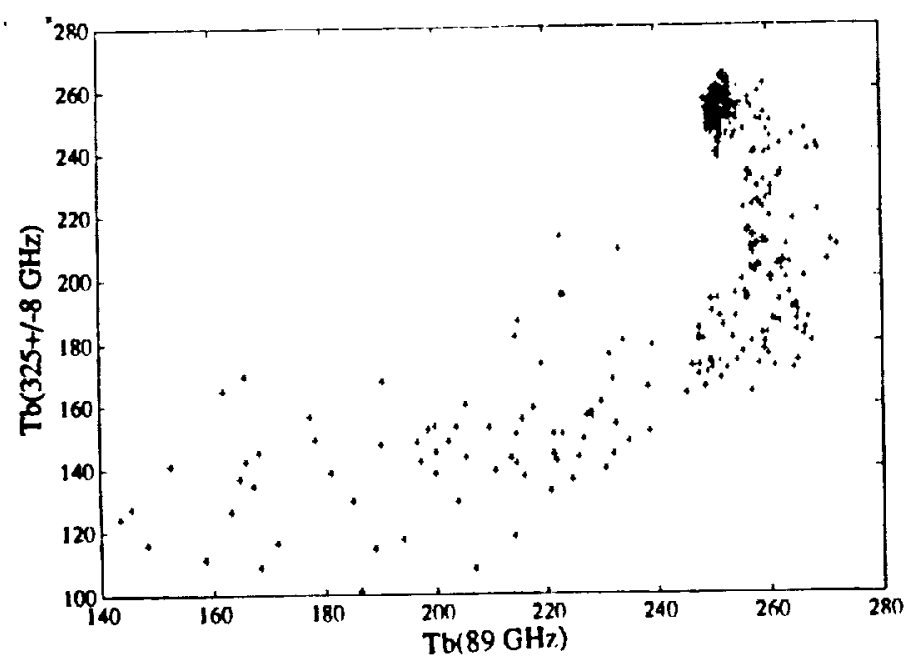

Figure 2: Scatterplot of nadir brightness temperatures over oceanic convection for the MIR 89 - and $325 \pm 8-\mathrm{GHz}$ channels (September 26, 1994).

window-channel brightness variations, the parameter most directly related to these variations is the vertically integrated ice content (IIC) in the cell top. The IIC is, in turn, highly correlated with the reflectivity of the cell top, and hence, the upwclling brightness temperature. Statistical retrieval of IIC from MMW observations at frequencies up to $\sim 90 \mathrm{GHz}$ using the dominant spectral modes of the raincell brightness temperatures appears feasible, although IIC retrieval algorithms are difficult to validate from field experiments.

The high sensitivity of the MIR's MMW and SMMW chan nels to IIC are expecled to further facilitate such IIC estimates. As shown by the nadir lineplot (Fig 3) the MIR window channels at $89,150,220$, and $325 \pm 8 \mathrm{GHz}$ all provide large responses to the ice canopies over raincells. The strength of these responses can be analyzed using the Karhunen-Loeve (KL) transform (Gasiewski and Staelin, 1989), computed as:

$$
\bar{k}=\overline{\bar{E}}^{\ell} \Delta \bar{T}_{B}
$$

where $\bar{E}$ is an orthogonal column matrix containing the eigenvectors of the brightness temperature covariance matrix and $\Delta \bar{T}_{B}$ is the brightness temperature referenced to clear air. From the data, the dominant spectral mode is found to have close to the same contributions from all four channels, indicating that these channels exhibit comparable variance from ice. This mode has a signal-to-noise ratio (SNR) greater than $\sim 35 \mathrm{~dB}$ for the MIR rain cell observations in Fig 3 .

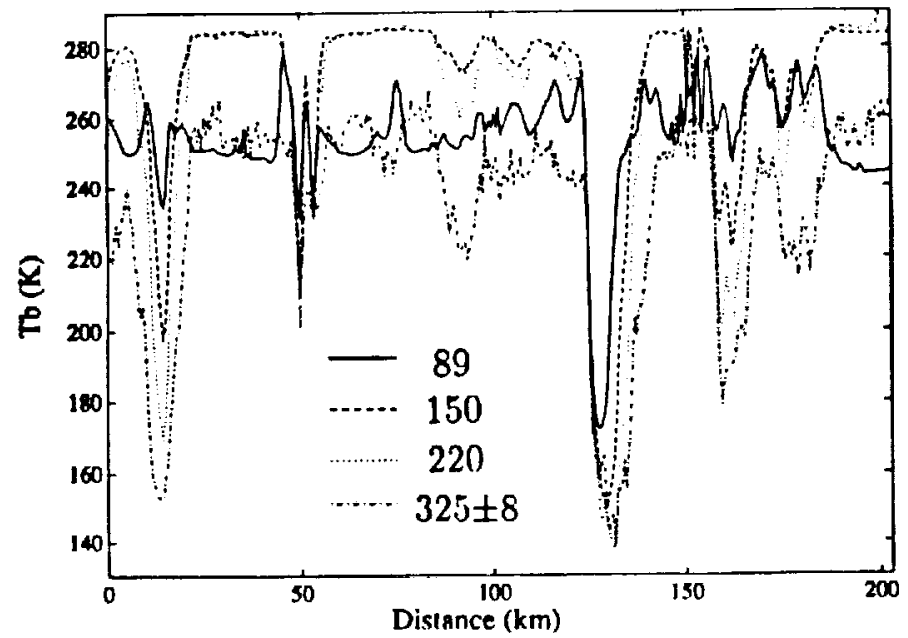

Figure 3: Brightness temperatures observed at nadir for the four MIR window channels at $89,150,220$, and $325 \pm 8 \mathrm{GHz}$. Plots are for the same overflight as in Fig. 1.

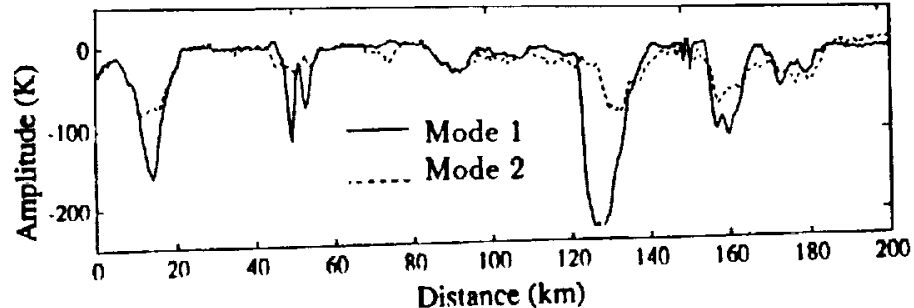

Figure 4: Two most dominant KL spectral modes for nadir MIR window channel observations over raincells. Plots are for the same overflight as in Fig. 1.

However, there are at least two other modes in the window channel data that exhibit SNR's greater than $10 \mathrm{~dB}$. The second most energetic mode is the difference between the high- and lowfrequency window channels; thus, it filters the slope of the raincell spectrum. This mode varies independently of the first mode as seen in Fig. 4 between 120 and $140 \mathrm{~km}$. Although meaning $f u l$ relationships between the KL modes and physical raincell parameters are not guaranteed, it appears that the second KL mode responds to radiometrically thin portions of the cell, in particular, the anvil regions. Indeed, because of the relatively flat spectrum of hydrometeor absorption and scattering, it is desirable to observe raincells using several channels that are widely separated in frequency. The MIR channels at $89,150,220$, and $325 \pm 8 \mathrm{GHz}$ provide such wideband measurements, spanning nearly two octaves in frequency.

\section{CONCLUSIONS}

The MIR data obtained during CAMEX show that simultaneous use of the two water vapor bands near 183 and $325 \mathrm{GHz}$ offers a viable means of both improving spaceborne passive measurements of cloud water content and of discriminating between clouds and waler vapor in passive retrievals. The $325 \cdot \mathrm{GHz}$ channels alone show potential for cloud and midlatitude raincell mapping without saturation or excessive water vapor screening. These channels are expected to be useful for high-resolution cloud parameter estimation and provide an improved means of monitoring global cloud water content. When used in conjunction with other MMW and SMMW window channels, the $325 \cdot \mathrm{GHz}$ channels provide unique information on the hydrometeor distribution through additional spectral degrees of freedom. While this initial analysis appears very promising, it is noted that the observations were performed only under summer midlatitude conditions. Additional observations are desirable under a variety of baseline meteorological conditions for a complete characterization of the available MMW and SMMW channels.

\section{ACKNOWLEDGEMENTS}

The authors would like to thank Dr. Ramesh Kakar at NASA Headquarters for his supporl of the MIR, and Scolt J. Sharpe at the Georgia Institute of Tochnology for assistance in the MIR dala processing. This work was supported by NASA grant NAG 5-1490 and the Georgia Tech Research Corporation.

\section{REFERENCES}

Gasiewski, A.J., and D.H. Staelin, "Statistical Precipitation Cell Parameter Estimation Using Passive 118-GHz $\mathrm{O}_{2}$ Observations," J. Geophys. Res., vol. 94, no. D15, pp. 18367-18378, December, 1989 .

Gasiewski, A.J., and D.H. Staelin, "Numerical Modelling of Passive Microwave $\mathrm{O}_{2}$ Observations Over Precipitation," Radio Sci., vol. 25 , no. 3, pp. 217-235, 1990.

Gasiewski, A.J., "Numerical Sensitivity Analysis of Passive EHF and SMMW Channels to Tropospheric Water Vapor, Clouds, and Precipitation," IEEE Trans. Geosci. Remote Sensing, vol. 30, no. 5, pp. 859-870, September 1992.

Racetle, P.E., R.F. Adler, A.J. Gasiewski, D.M. Jackson, J.R. Wang, and D.S. Zacharias, "An Airborne Millimeter-Wave Imaging Radiometer for Cloud, Precipitation, and Atmospheric Water Vapor Studies," submitted to J. Atmos. Oceanic Tech., February 1994 . 